\title{
Article \\ Computational Algorithms for the Study of Distributions with Electric Charge and Radiation Flux in General Relativity
}

\author{
Franyelit Suárez-Carreño ${ }^{1, *(1)}$ and Luis Rosales-Romero ${ }^{2}$ \\ 1 Facultad de Ingeniería y Ciencias Aplicadas, Carrera de Ingeniería Industrial, \\ Universidad de las Américas (UDLA), Quito 170902, Ecuador \\ 2 Departamento de Ciencias Básicas, Universidad Politécnica de Venezuela, UNEXPO, \\ Puerto Ordaz 8050, Venezuela; lrosales@unexpo.edu.ve \\ * Correspondence: franyelit.suarez@udla.edu.ec
}

check for

updates

Citation: Suárez-Carreño, F.; Rosales-Romero, L. Computational Algorithms for the Study of Distributions with Electric Charge and Radiation Flux in General Relativity. Appl. Sci. 2021, 11, 5957. https://doi.org/10.3390/ app11135957

Academic Editor: Alexander N. Pisarchik

Received: 23 May 2021

Accepted: 10 June 2021

Published: 26 June 2021

Publisher's Note: MDPI stays neutral with regard to jurisdictional claims in published maps and institutional affiliations.

Copyright: (c) 2021 by the authors. Licensee MDPI, Basel, Switzerland. This article is an open access article distributed under the terms and conditions of the Creative Commons Attribution (CC BY) license (https:/ / creativecommons.org/licenses/by/ $4.0 /)$.

\begin{abstract}
The following research contributes to the understanding of how electric charge influences the unwrapping of spherically symmetric distributions using a relativistic approach. With the recent detection of gravitational waves and the location of black holes, where a strong emission of electromagnetic radiation prevails, interest has arisen to consider the existence of compact charged objects. Thus, the appearance of charge in small quantities during gravitational collapse, the process by which black holes originate, is not ruled out; this also includes the emission of electromagnetic waves from them. This article intends to establish algorithms and write field equations for a charged fluid as those corresponding to an anisotropic fluid with radiation flux. Using an appropriate definition of the mass function, considering self-similar symmetry and Bondian observers, dynamical results are obtained for the Einstein-Maxwell electromagnetic system with added gravity. Imposing an additional homothetic symmetry, the field equations are solved, and the most relevant conclusions are drawn about the influence of the electric charge during the collapse and subsequent changes in the physical variables.
\end{abstract}

Keywords: einstein field equations; electric charge; algorithms

\section{On the Referential Theory of Research Review of the Field Equations Posed by Einstein}

Field equations developed by Einstein, which form the basis of the theory of general relativity (GR), relate the geometry of space-time (Einstein Tensor) to the distribution of matter (energy-momentum tensor) (Equation (1))

$$
G_{\mu v}=-8 \pi T_{\mu v}
$$

GR replaced the theory of universal gravitation by explaining effects that cannot be accounted for by Newton's laws, such as the anomaly in the orbit of Mercury and other planets. It also makes numerous predictions, some already formed about the effects of gravity, such as the bending of light and time contraction. In addition, it predicts space-time distortions, a phenomenon known as gravitational waves.

The study of a system composed of a radiating fluid is possible through these equations. The solution of these equations allows, on the one hand, to obtain a set of physical variables, density, pressure, fluid velocity, and energy flow, and, on the other hand, information about the geometrical structure of space-time. Following a literature-based approach, the problem can be analyzed by considering that the surface of the distribution separates space-time into two different sections: the section inside the distribution, referred to as the inner zone, where there are high concentrations of both matter and energy, and the section outside the surface, referred to as the outer zone, with radiation freely escaping to infinity. It is, therefore, of great importance to conceptualize the inner and outer regions 
properly from a geometrical point of view, as well as the characteristics of their energetic material content.

Some physical characteristics of collapsing charged matter distributions may justify the interest in considering whether the electric charge affects gravitational collapse. For example, at certain times of collapse, electric charge in reduced amounts can change the final state of an astrophysical object.

The study of spherically symmetric charged distributions begins with Eddington [1], who showed that, if an isolated point charge has an oscillatory potential, the Lorentz gauge [2] allows all components of the metric to be radiative, even if they are static. Although some refer to static situations [3], significant efforts have been made to describe dynamic situations. Recently, great interest has arisen in studying the effect of large amounts of charge in spherically symmetric systems, although by an approximation in pre-relativistic physics it can be proved that a physical object possesses a quantity of charge greater than one hundred coulomb over $\mathrm{M}_{\Theta}$ (solar mass) [4].

It is important to highlight the possibility that strong electric fields may exist in strange stars [5]. Some authors [6] describe models of collapsing newly charged spheres including dissipative processes and emphasize the importance of electric charge in the interactions present in these systems. Specifically, they show that the electric charge does not cause regeneration effects (it does not add more activity to the present field).

To solve problems in GR, it is necessary to introduce certain initial conditions, such as thermodynamic principles, a correlation between measurable variables, additional symmetries to space-time [7-10], or to use the post-quasistatic approach. For example, Ray et al. [11] study charged fluid spheres in an Einstein-Maxwell space-time under the imposition of conformal symmetry. In a study on relativistic stars [12], it was observed that the event horizon occurs when the radius reaches its limit; it is at that time that the gravitational attraction can be balanced in the presence of a large amount of electric charge $[2,13,14]$. Bonnor and Wikramasuriya [15] investigated the effects of electric charge on the matter, whose electrostatic repulsive force balances the gravitational attractive force $[15,16]$.

Other research on electric charge includes that of Barreto and Da Silva $[13,14]$, who study self-similar charged spherical distributions in the diffusion approximation. Ray et al. [11] use classical arguments to check the electric neutrality of stars and show that, if the pressure, density, and gravitational field are large, it means that high values of charge and intense electric fields could exist in such distributions. Ray et al. [11] analyze the influence of electric charge on compact objects (in that case, stars) and observe how the equilibrium of forces allows a charge in them of about $10^{20}$ coulomb and electric fields around $10^{20}$ coulomb and electric fields around $10^{21} \mathrm{~V} / \mathrm{cm}[11,17,18]$.

Barreto et al. $[17,18]$ noted that solving the differential equations defining these problems can be used to find the physical characteristics of collapsing stars. These studies show that the exact solutions of electromagnetic systems with coupled gravity are important in the representation of compact objects, such as quark stars or preon stars. Some researchers [19] found solutions to these systems where charged spheres are present with a specific type of field, both electric and gravitational. These researchers [20] showed the solutions they obtained for these types of arrangements where the electric and gravitational fields have a specific shape.

In studies of compact relativistic objects, strange stars have been proposed as a different option to the well-known study of neutron stars, and anisotropy (intrinsic, electric charge-induced, or by any other mechanism) plays an important role when analyzing these objects. If the anisotropy is induced by electric charge, naturally, the Tolman-OppenheimerVolkoff Equation (1) [21] should be modified to consider the charge within the distribution. Among other possibilities, one of the reasons for considering anisotropy within stars could be the presence of strong electric fields as recently suggested by Usov [5].

Among other works related to electric charge, we can mention that of Rosales et al. [17], where they study static fluid spheres in isotropic coordinates and find a central equation of 
state for the distribution. Ivanov [15] demonstrates the relationship between anisotropy and charged fluids.

A comprehensive study of the influence of electric charge on anisotropic strange star aspirants takes into account an interior measure of space-time with spherical symmetry. To solve the resulting field equations, it is observed that the cumulative electric charge distribution is maximal over the surface possessing an estimated electric charge in the range of $10^{20}$ coulomb and an electric field in the range of $10^{21} \mathrm{~V} / \mathrm{cm}$ a $10^{22} \mathrm{~V} / \mathrm{cm}$ [22].

In $[17,18]$, new ways were obtained to solve the field equations where matter possesses both anisotropy and electric charge. When a spherically symmetric and static space-time is assumed, an equation of state of quadratic type (Equation (2)) is used to know the different gravitational potentials and the electric field strength, through which the abovementioned new exact solutions for isotropic graphical charts are obtained. In $[13,14,16,18]$, an algorithmic method was disclosed that could model the behavior of all fluid distributions possessing charge with spherical symmetry, considering other different function, and a set of physically acceptable solutions was found, and representative compact stellar models were reported.

$$
p_{r 0}=\eta\left(\frac{3 a b}{2 \pi}\right)^{2}+\alpha\left(\frac{3 a b}{2 \pi}\right)-\beta
$$

The study of a charged compact object with anisotropic pressures and core governed by $a$ quadratic equation of state was analyzed in [23], and it was concluded that the masses, radio, and other compactness factors of stellar objects are affected by the presence of an electric field when these values agree with those of previous studies [24].

This work attempts to delve into the study of charged, radiative, and spherically symmetric fluids. An important aspect to consider is that the question of whether charged relativistic spheres form black holes remains unanswered. In this work, we make an interesting contribution in this sense, solving the equations of the governing electromagnetic systems that also include gravity (Einstein-Maxwell systems) and considering both the equation describing the conservation of charge and the Tolman-Oppenheimer-Volkov equation [21].

\section{Development}

\subsection{The Metric, Energy-Momentum Tensor, and Field Equations}

The metric for defining the shape of the distribution on which the profiles of geometrical and physical variables in general relativity will be evaluated; the choice of a suitable coordinate system greatly simplifies the analysis of the system under study. In particular, in the study of radioactive systems, the description provided by the so-called radiation coordinates or Bondi metrics [25] is adequate. Therefore, for the description of the geometry of the interior of the distribution, the Bondi metrics [25] are used. Considering a spherically symmetric matter distribution, which is given by a charged fluid, with values of pressure, energy, electric energy density, and radiation energy flux given by $p, \rho, Q$, and $\epsilon$, respectively, and measured from the perspective of an observer in a Minkowski representation moving at par (comoving) with the fluid (with velocity $-\omega$ ), the metric is expressed in a radiation coordinate system [26-28] as Equation (3):

$$
d s^{2}=e^{2 \beta}\left(\frac{V}{r} d u^{2}+2 d u d r\right)-r^{2}\left(d \theta^{2}+\sin ^{2} \theta d \phi^{2}\right)
$$

of which $\beta$ is a function $u$, and $V$ is a function of $r$, the Bondi time being $u=x^{0}$ [25] at null infinity, where $u$ is constant. Where $r=x^{1}$ is the radial coordinate tangent to the null cones. Moreover, both $\theta=x^{2}$ as well as $\varphi=x^{3}$ correspond to angular coordinates. Geometric units will be used throughout this work. $G=c=1$. From the metric function $V$, the function $m$ $(u, r)$ is defined, which represents the mass term in the interior of the distribution. This quantity was introduced by Bondi [29] as a generalization of the mass in the static case 
to the radiation case and is related to $\beta$ and for the case of charged distributions by the following expression:

$$
V=e^{2 \beta}\left(r-2 m\left(1-\frac{2 C}{r}\right)\right)
$$

where $C$ represents the charge inside the distribution. Knowing that, in spherical symmetry, the only components with nonzero values of the electromagnetic field tensor are $F^{u r}=-F^{r u}$ (Equation (4)). Then, a function $C(u, r)$ can be defined by the correspondence observed in [30-32].

$$
F=\frac{C}{r^{2}} e^{-2 \beta}
$$

The nonhomogeneous Maxwell equations are Equations (6) and (7)

$$
\begin{gathered}
C_{, r}=4 \pi r^{2} J^{u} e^{2 \beta} \\
\text { and } C_{, u}=-4 \pi r^{2} J^{r} e^{2 \beta}
\end{gathered}
$$

The partial derivative as a function of the indicated coordinate is denoted by the comma symbol (,); then, the time component is represented by $J^{u}$ and the radial component by $J^{r}$, both belonging to the electric current density quadrivector; then, the idea is that $C(u, r)$ shows a function of charge at radius $r$ at a given time $u$. In this way, the conservation of charge in the inhomogeneous electromagnetic equations is expressed as well as observed in Equation (8)

$$
U^{, \mu} C_{\mu}=0
$$

where the quadravelocity of the fluid is given by (9).

$$
U^{\mu}=e^{-\beta}\left[\sqrt{\frac{r}{V}}\left(\frac{1-\omega}{1+\omega}\right) \delta_{u}^{\mu}+\sqrt{\frac{V}{r}} \frac{\omega}{\left(1-\omega^{2}\right)^{1 / 2}} \delta_{r}^{\mu}\right]
$$

\subsubsection{Energy-Momentum Tensor}

We now follow the approach of Bondi [29-32], reported as Bondian observers of the GR [9]: a local observer is located, according to the Minkowski perspective, in motion with the fluid charged with velocity $-\omega$; for such an observer following the radial direction (the components of the energy-momentum tensor are given by the energy density, the electric energy density, the unpolarized energy density, and the pressure represented by the symbols $\rho, \mu, Q$, and $p$, respectively), Equation (10) will represent the covariant energy-momentum tensor.

$$
T_{v \mu}=(\rho+p) u_{\mu} u_{v}-p g_{\mu v}+\epsilon l_{\mu} l_{v}+E_{\mu v}
$$

where density $\rho$, pressure $p$, metric tensor components $g_{\mu v}$, radiation flux $\epsilon$, null vector $l_{\mu}$, and electromagnetic tensor $E_{\mu v}$ are identified. For this paper, it is more useful to write Equation (10) as shown in Equation (11).

$$
T_{\mu v}=\left(\begin{array}{ccccc}
\hat{\rho}+\hat{\mu}+\hat{\epsilon} & & -\hat{\epsilon} & 0 & 0 \\
-\hat{\epsilon} & & \hat{\rho}+\hat{\mu}+\hat{\epsilon} & 0 & 0 \\
& 0 & 0 & \hat{\rho}+\hat{\mu} & 0 \\
& 0 & 0 & 0 & \hat{\rho}+\hat{\mu}
\end{array}\right)
$$

where they are identified as density $\hat{\rho}$ and effective pressure $\hat{p}$, respectively, and $\hat{\mu}=E^{2} / 8$, with the electric field given by $E=C / r^{2}$ and $p_{t}=p+\hat{\mu}$. As mentioned above, $C(u, r)$, is the function representing the charge for any $r$ at time instant $u$ and satisfies the conservation of charge described in Equation (12) for numerical purposes.

$$
C_{, u}+\frac{d r}{d u} C_{, r}=0
$$


As shown above, the comma (,) is the partial derivative concerning the indicated coordinate. Equation (13) represents the velocity of matter.

$$
\frac{d r}{d u}=\frac{V}{r} \frac{\omega}{1-\omega}
$$

When the fluid is adiabatic, it possesses a diagonal energy tensor in covariant form $\left(\rho, p_{r}, p_{t}, p_{t}\right)$ defining $\hat{\rho}=\rho+\hat{\mu}, p_{r}=p-\hat{\mu}$, which is the same for an anisotropic fluid $[16,17]$. It is clear that, in the presence of electric charge, local anisotropy occurs. On the contrary, in the absence of electric charge, the Pascalian character of matter, i.e., isotropic fluid, is recovered. The variables $P_{t}$ and $P_{r}$ are the tangential and radial pressures, respectively.

In addition, by defining the mass function as shown in Equation (14),

$$
m=\frac{1}{2}\left(r-V e^{2 \beta}\right)
$$

the so-called Missner-Sharp mass generalization $[33,34]$ is obtained. When there is charge, it is expressed as

$$
m=m+\frac{C^{2}}{2 r}
$$

\subsubsection{Field Equations}

After several algebraic manipulations, the field Equation (1), is written according to Equations (16)-(19) [15,16].

$$
\begin{gathered}
\frac{\rho+p \omega^{2}}{1-\omega^{2}}+\frac{1+\omega}{1-\omega} \hat{\epsilon}=\frac{e^{-2 / 3}\left(C C_{, u} / r-m_{, u}\right)}{4 \pi\left(r-2 m+C^{2} / r\right)}+\frac{m_{, r}-C C_{, r} / r}{4 \pi r^{2}} \\
\frac{\rho-p \omega}{1+\omega}=\frac{m_{, r}-C C_{, r} / r}{4 \pi r^{2}} \\
\frac{(1-\omega)}{1+\omega}(p+\rho)=\frac{\beta_{, r}}{2 \pi r^{2}}\left(r-2 m+C^{2} / r\right) \\
p=-\frac{1}{4 \pi} \beta_{, u r} e^{-2 \beta}+\frac{1}{8 \pi}\left(1-\frac{2 m}{r}+\frac{C^{2}}{r^{2}}\right)\left(2 \beta_{, r r}+4 \beta_{, r}^{2}-\frac{\beta_{, r}}{r}\right) \\
+\frac{1}{8 \pi r}\left[3 \beta_{, r}\left(1-2 m_{, r}\right)-m_{, r r}\right]+\frac{3 \beta_{, r}}{8 \pi r}\left(2 C C_{, r} / r-C^{2} / r^{2}\right) \\
+\frac{1}{8 \pi r^{2}}\left(C_{, r}^{2}+C C_{, r r}-2 C C_{, r} / r\right)
\end{gathered}
$$

Making adjustments, $\hat{\rho}=\rho+\epsilon, p_{r}=p-\hat{\mu}, \hat{\mu}=E^{2} / 8 \pi$, with $E=\frac{C}{r^{2}} y$ $p_{t}=p+\hat{\mu}$. The field equations can be written as Equations (20)-(23).

$$
\begin{gathered}
\frac{\rho+p_{r} \omega^{2}}{1-\omega^{2}}+\hat{\epsilon} \frac{(1+\omega)}{1-\omega}=-\frac{e^{-2 \beta} m_{, u}}{4 \pi r(r-2 \mu)}+\frac{m_{, r}}{4 \pi r^{2}} \\
\widetilde{\rho}=\frac{m_{, r}}{4 \pi r^{2}} \\
\widetilde{\rho}+\widetilde{p}=\frac{\beta_{, r}}{2 \pi r^{2}}(r-2 m) \\
p_{t}=-\frac{1}{4 \pi} \beta_{, u r} e^{-2 \beta}+\frac{1}{8 \pi}(1-2 m / r)\left(2 \beta, r r+4 \beta_{, r}^{2}-\beta, r / r+\frac{1}{8 \pi r}\left[3 \beta_{, r}\left(1-2 m_{, r}\right)-m,\right]\right)
\end{gathered}
$$$$
\widetilde{p}=\frac{p_{r}-\omega \rho}{1+\omega}
$$

where

and

$$
\frac{\hat{\rho}-\omega p_{r}}{1+\omega}
$$


By knowing the metric $\beta(u, r)$ and the metric $m(u, r)$, together with their derivatives, it is possible to determine $p, \rho, \epsilon$, and $\omega$ from the symmetry equations. Equations (20)-(23) are the same for anisotropic matter [18,33-35], so that it can be interpreted as anisotropy of the fluid, to the electric charge. Such an interpretation is possible because of how the mass (Equation (14)) is defined, so that the electric charge can be assumed as a constituent of the metric. Assuming this position, the electric charge would then become another physical variable that contributes to both energy density and pressure. This model helps with the understanding of what has been studied due to the approximation of the Bondian observers to the GR (comoving frame of reference). Barreto et al. [32,36] showed that the observers used by Bondi for the treatment of matter in GR and those used by the standard ADM $3+1$ of numerical relativity are the same, both have comoving and Minkowskian-type observers, as mentioned above. These observers are the same as those of the post-quasistatic approximation of GR for the study of collapsing spheres. The treatment is identical for an intrinsic anisotropic fluid, except for the mass function.

At this point, there are four field equations for seven unknowns: two geometric variables $m$ and $\beta$ and five physical variables $p$, rho, radiation flux, velocity, and charge.

The solution algorithm is as follows:

- The geometric variables are obtained from the imposition of an additional homothetic (self-similar) symmetry, i.e., from the symmetry equations as a function of the surface variables.

- $\quad$ Previously, to find the surface variables, the surface equations must be solved by the Runge-Kutta method of order 4 [36].

- $\quad$ The electric charge is determined from the numerical solution of the charge conservation equation.

- $\quad$ Pressure, density, radiation flux, and velocity are determined from the field equations.

\section{Results}

The case study of a system composed of a radiant fluid with electric charge is possible through the field equations describing the Einstein-Maxwell system. The solution of these equations allows, on the one hand, for obtaining a set of physical variables, density, pressure, fluid velocity, energy flow, and electric charge, and, on the other hand, information about the geometrical structure of space-time. As already mentioned, in studying this problem, it is necessary, according to the theoretical approach, that space-time is divided in these configurations by the surface of the distribution into two regions: an inner one containing matter and radiation and an outer one where radiation escapes if difficulty of the system moves toward infinity. It is therefore necessary to show the geometry that defines both the inner and outer regions, as well as to characterize their energetic material content.

\subsection{Reissner-Nordstrom-Vaidya Space-Time}

The spherically symmetric charged distribution is considered to be bordered by the surface $\sum$. Whatever is not contained in it belongs to an RNV-type space-time described in Equation (26), according to [16,17].

$$
d s^{2}=\left(1-2 M(u) / r+C^{2} / r^{2}\right) d u^{2}+2 d u d r-r^{2}\left(d \theta^{2}+\operatorname{sen} \theta^{2} d \varphi\right)
$$

In Equation (26), the total mass is given by $M(u)$, and the total charge is $C$. Smoothly coupling Equations (2) and (26) on the hypersuperciliation $r=\sum$.

$$
m(u, \Sigma)=m(u) \beta(u, \Sigma)=0 p \Sigma=0 \mathrm{Q} \Sigma=\mathrm{C}
$$

Once this moment is reached, which is the Bondi approximation for spherical symmetry, the equations obtained are of a general type. We identify five variables $(\rho, p, \omega, \epsilon, C)$ that are physical, plus two $(m, \beta)$ metric functions and four field equations in addition to the conservation equation. Other considerations need to be added in order to solve the 
characteristic initial value problem. Under these circumstances, space-time is assumed to be self-similar, which will allow us to illustrate how the transport mechanism influences the evolution of charged spheres.

\subsection{Self-Similarity and Surface Equations}

The inner zone, characterized by Equation (2) and the outer zone, described by the Reissner-Nordstrom-Vaidya metric [16,17], are separated by the hypersurface $r=\sum$. Therefore, the coupling of the inner and outer solutions of the field equations must be considered. Furthermore, to know the evolution of the physical variables in the interior of the distribution $(p, \rho, C, \epsilon, \omega)$ in order to evaluate quantities over the surface, it is necessary to construct a system of ordinary differential equations relevant.

Having done the above, a system of three ordinary differential equations is obtained, with three functions of time $\mu$ associated with the radius of the distribution, the velocity at the edge of the distribution, and the function $m$ evaluated at the surface.

Some authors [37] characterized covariantly the self-similarity when the matter is distributed spherically, noting that the self-similar solutions depend on whether they are invariant or not in the presence of scale transformations. Seen mathematically, the interesting thing about self-similarity is that it helps to reduce the field equations to a group of ordinary differential equations, and, on the other hand, because it helps to generalize the symmetries, homothetic, and Killing symmetry, according to [38-40].

To define self-similarity requires the existence of a homothetic Killing vector field. In a given coordinate system, self-similarity is evidenced by a simple scaling relation for the metric functions. Probably no problem in GR has been more exhaustively studied than that of spherically symmetric homothetic space-times [37].

Now, an additional homothetic (self-similar) symmetry is imposed, which mathematically is expressed in Equation (28) [16-18].

$$
\mathcal{L}_{\xi} g_{\mu v}=2 g_{\mu v}
$$

where $g_{\mu v}$ is the metric tensor, and the homothecal generator $\xi$ is expressed as

$$
\xi^{\mu}=\Lambda(u, r) \delta^{\mu}+\lambda(u, r) \delta^{\mu}
$$

Putting Equations (28) and (29) together with (22) leads to

$$
\Lambda Y_{u}+r Y_{r}=0
$$

and

$$
X_{u}+r X_{r}=0
$$

which can also be written as

$$
\begin{aligned}
& \zeta(X(u, r))=0 \\
& \zeta(Y(u, r))=0
\end{aligned}
$$

By solving Equations (30) and (31), it is possible to know the form of the metric variables $\beta$ and $m$ for all $Y(u, r)$.

\subsection{Proposed Solution to the Problem}

When a problem in classical mechanics is very symmetric, it is required to simplify the partial differential equations to ordinary differential equations, where the dependent variables behave in essence as a single variable, which depends on the unstressed combination of the independent variables. Thus, when a problem has spherical symmetry, the independent variables are the radius at the center of symmetry $r$ and the time $t$, and the dependent variables are a function of Equation $(34)[16,17]$.

$$
\mathcal{Z}=\frac{r}{r_{0}}\left(\frac{t}{t_{0}}\right)^{-n} \zeta
$$


This method has been successfully employed in the nonrelativistic theory of selfgravitating fluids. This technique will be applied to Equations (30) and (31).

If new independent variables $\mu$ and $r$ are defined, then $X=X(\zeta)$ and $Y=Y(\zeta)$ are solutions of the Equations (32) and (33) when the self-similar variable is expressed as

$$
\zeta=r e^{-\int \frac{d u}{\Lambda}}
$$

Defined, $Y=\frac{\Lambda e^{2 \beta}}{r}$ and $X=\frac{m}{r}$. The solution of Equations (32) and (33) is of the form

$$
Y=C_{1} r^{k} e^{-k \int \frac{d u}{\Lambda}}
$$

and, making use of differential geometry for the continuity of I (first fundamental form), there are solutions for $m$ and $\beta$, which are

$$
e^{2 \beta(u, r)}=\left(\frac{r}{r_{\Sigma}}\right)^{l+1}
$$

and

$$
m(u, r)=m_{\Sigma}\left(\frac{r}{r_{\Sigma}}\right)^{k+1}
$$

In the above equations, the values of $k$ and $l$ are constants, and the quantity is evaluated on the surface $\Sigma$, as indicated by the subscript, such that $r_{\Sigma}(\mu)$ represents the radius of the distribution.

\subsection{Equations on the Surface. Coupling Conditions}

In general relativity, there are coupling conditions known as Darmois conditions [41]. The ultimate purpose of these conditions is to avoid the appearance of singular behavior of physical variables on the coupling surface [18,19]. According to Darmois [41], the necessary and sufficient condition for the coupling of two space-time regions on a hypersurface is that both the metrics induced on it (first fundamental form).

The inner region, characterized by the line element and the outer region, described by the Reissner-Nordstrom-Vaidya metric [16], are separated by the hypersurface $r=\Sigma(\mu)$. Therefore, the coupling of the inner and outer solutions of the field equations must be considered. Furthermore, to know the evolution of the physical variables inside the distribution, it is necessary to construct a system of ordinary differential equations for quantities evaluated on the surface. To obtain these equations, we proceed as follows:

- The functions $m$ and $\beta$ are required to be continuous across the edge of the distribution.

- The material velocity at the surface is evaluated.

- The first field Equation (20) is evaluated on both sides of the surface.

- $\quad$ The conservation equation is calculated $T_{, 1 ; \mu}^{\mu}=0$.

The energy conservation equation leads to

$$
\begin{gathered}
\frac{\partial p}{\partial r}+\frac{\bar{\rho}+\bar{p}}{1-2 \mu / r}\left[4 \pi r \bar{p}+\mu / r^{2}\right]-e^{-2 \beta}\left(\frac{\bar{\rho}+\bar{p}}{1-2 \mu / r}\right)_{, u}=\frac{2}{r}\left(p_{t}-\bar{p}\right) \\
-\beta_{, u} e^{-2 \beta}+\left(1-\frac{2 m}{r}\right) \beta_{, r}-\frac{m_{, r}}{2 r}+\frac{C^{2} T}{4 r^{3}}=0
\end{gathered}
$$

evaluated on the $\sum$ surface.

Once the above is done, two ordinary differential equations are obtained with two functions of time to be determined, corresponding to

- The velocity of the edge of the distribution.

- The function $r=\Sigma$. 
These are the surface radius $r_{\Sigma}$, the distribution edge velocity, and the function evaluated on $r=\Sigma$. It is noticeable that the metric functions and, thus, the electric charge and physical variables are obtained from the time dependence of the variables at the surface $m_{\Sigma} \mathrm{y} r_{\Sigma}$, for which the differential equations on the surface are required, which are obtained from Equations (40) and (41) evaluated at $\Sigma$

$$
\begin{gathered}
\dot{m}_{\Sigma}=-\epsilon_{\Sigma}\left(1-\frac{2 m_{\Sigma}}{r_{\Sigma}}\right)+\dot{r}_{\Sigma} \frac{q^{2}}{2 r_{\Sigma}^{2}} \\
\dot{r}_{\Sigma}=\left(1-\frac{2 m}{r_{\Sigma}}\right) \frac{\omega_{\Sigma}}{1-\omega_{\Sigma}}
\end{gathered}
$$

where the point on the variable indicates time derivative. In addition, the radiation flux at the surface:

$$
\epsilon_{\Sigma}=\hat{e}\left(1+\omega_{\Sigma}\right) /\left(1-\omega_{\Sigma}\right)
$$

The field Equation (22) evaluated on the surface gets to know the total mass of the distribution of matter

$$
m_{\Sigma}=\frac{1}{\psi}\left(\xi r_{\Sigma}-\frac{C^{2}}{r_{\Sigma}}\right)
$$

where $\xi=(l+1)^{2}$ and $\psi=2 \xi+k(4+3 l+k)$. This allows us to obtain $\epsilon_{\Sigma}$ from Equations (22) and (23) and the surface equations.

$$
\epsilon=\left\{\frac{C^{2}}{r_{\Sigma}^{2}}\left[\frac{1}{2}+\frac{1}{\psi}\right]-\frac{\xi}{\psi}\right\} \frac{\omega_{\Sigma}}{1-\omega_{\Sigma}}
$$

Such that one has a single equation on the surface that is numerically integrated with the specification of the total mass $m_{\Sigma}$ and the radius of the distribution $r_{\Sigma}=\mathrm{R}$ and the radius of the distribution $r \Sigma=\mathrm{R}$. Once the dynamics on $\Sigma$ have been obtained, the following procedure is followed to calculate the values of the physical variables:

- Specifying the initial electric charge distribution allows for finding the physical variables at any radius (or any layer) of the sphere.

- Integrate Equation (3) to advance the charge $C$ in time.

- We have the evolution of the physical variables for all time and all radius within the matter distribution.

Below is the routine in FORTRAN 77 for the solution of the surface equations with Runge-Kutta (Figure 1). 


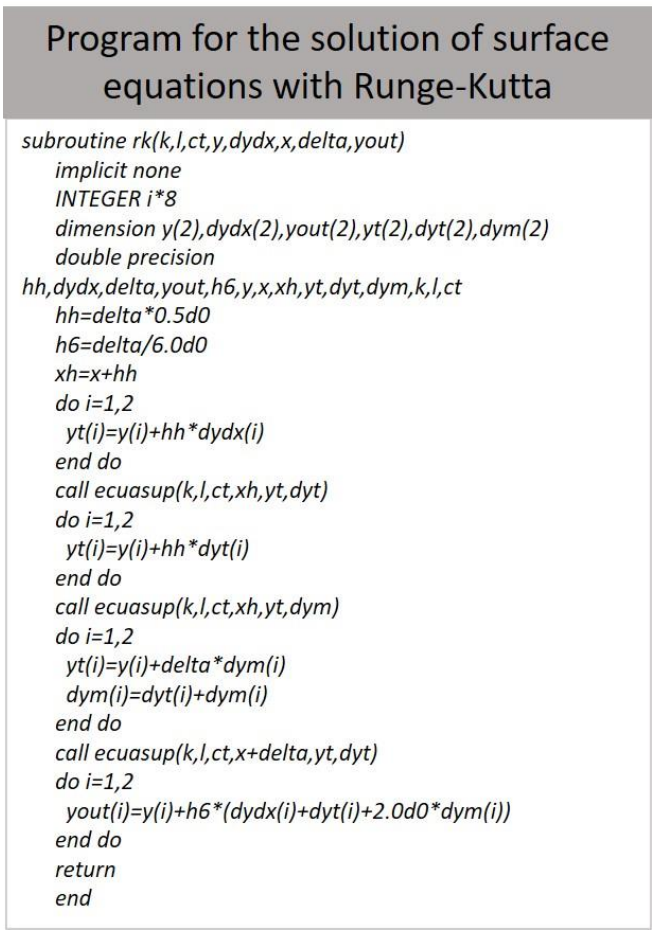

Figure 1. Solution of surface equations with Runge-Kutta.

\section{Discussion of Results}

A priori, to find solutions to electromagnetic systems with the presence of gravity (Einstein-Maxwell systems) as those proposed in the present work, there are no restrictions in the specification of which values the constants $k, l$, and the initial charge $C$ can take. Only that the choice allows us to obtain physically acceptable behaviors, as illustrated in the results. Any choice of the above parameters must be consistent with $-1<w \Sigma<1$ and $m \Sigma>0$ over the initial light cone (integration cone).

At this point, we have a system of four nonlinear differential equations in partial derivatives, in which geometry (metric functions $m$ and $\beta$ with the material content of the system under study (pressure, density, electric charge, and radiation) and dynamics (velocity of matter in the comoving, $\omega$ ) are mixed. All these variables correspond to the interior of the distribution. The time evolution of the surface of the distribution, how it collapses, and the speed at which it collapses must also be known.

\section{Integration of the Conservation Equation}

Once the integration of the equations on the studied surface has been achieved, it is necessary to proceed in the same way with the conservation equation to know the values of all the physical variables within the source. For this purpose, the spatial marker of comovility $x=r / a$. is used. Thus, the conservation equation can be written in the following way:

$$
C_{, u}=-\frac{d x}{d u} C_{, x}
$$

which is defined as a wave equation that is integrated by using the numerical method of Lax-Friedrichs, with convergence conditions of Courant-Friedrichs-Levy (CFL). When treating the conservation equation in the described way and obtaining its solution, it must be remembered that this solution is conditioned by the evolution of the surface, so it was implemented as follows:

$$
C_{j}^{m+1}=\frac{1}{2}\left(C_{j+1}^{m}+C_{j-1}^{m}\right)+\frac{\delta u}{2 \delta x}\left(\frac{d x}{d y}\right)_{j}^{n}\left(C_{j+1}^{m}-C_{j-1}^{m}\right)
$$


In Equation (47), one has that the superscript $\mathrm{n}$ is associated with $\mu=n \delta \mu \mathrm{u}$, which is a hypersurface, and the subscript $\mathrm{j}$ is associated with the observer in comoving with $x=n \delta x$. Typically, $\delta \mu=10^{-2}$ with the CFL condition $\delta \mu=2 \delta x$. The initial and boundary conditions need to be defined in order to proceed to integrate the conservation equation. For the case study of this paper, the boundary condition is given by

$$
C(x=0, u)=0
$$

Adding the initial condition results in the following:

$$
C(x, 0)=C X^{p}
$$

where the power $\mathrm{p}$ made it possible to investigate the level of effect of the initial conditions on the obtained results. The initial charge function is specified (its radial dependence) so that it satisfies the conservation equation. At this point, there are no restrictions to go inside the distribution and find the time evolution of the physical variables. For this, the specific charge function in relation to the radial coordinate $r$, at the time $\mu=0$, needs to be determined. Taking into account whether the time evolution does not spoil the physical requirements, the models can be obtained without difficulty.

The sphere was divided into five layers, where the physical variables obtained from the field equations are evaluated. The collapse was imposed by imparting an initial velocity to the surface, which allows the evolution of the surface. The integration of the differential equations on the surface was done with the fourth-order Runge-Kutta method. In addition, the physical variables during the integration were kept bounded within physically acceptable values.

$$
\rho>0, P>\rho,-1<\omega<1
$$

This is density greater than zero, pressure greater than density, and velocity bounded between -1 y 1 , thus avoiding propagation velocities above the speed of light. This is why the CFL condition is used.

An important feature is the anisotropy factor $p_{r}-p_{t}=2 \hat{\mu}$, where it is evident that the anisotropy is induced by the charge. If the value of the charge is zero, of course, the anisotropy factor is zero. The radius of the distribution collapses inexorably. Unlike $[2,13,18]$, the electric charge does not stop the collapse. Figure 2 shows the FORTRAN 77 routine to solve numerically the charge conservation equation.

Figure 3 models the rate of change of the radius of the distribution over time, where it is observed that it collapses inexorably. In addition, the evolution of the physical variables is shown.

Figure 4 shows the evolution when $r / r_{\Sigma}$ varies from 0.6 to 1.0 , for $k=0.35 ; l=0.5$ initial charge and radius $C=0.292$ and $R(0)=2.923$, respectively.

Figure 5 shows the evolution of radial pressure with time, for $r / r_{\Sigma}$ from 0.6 to 1.0, for the values $k=0.35 ; l=0.5 ; C=0.292$; and $\mathrm{R}(0)=2.923$. The evolution is shown for different layers of the matter distribution.

Figure 6 shows the radiation flux (multiplied by 102) for $k=0.35 ; l=0.5$ and initial conditions $C=0.292$ and $R(0)=2.923$. The evolution with time for different layers of the matter distribution is shown.

Figure 7 shows the evolution of the charge function with time for constants $k=0.35$; $l=0.5$ and initial charge conditions and radius $C=0.292$ and $R(0)=2.923$. 

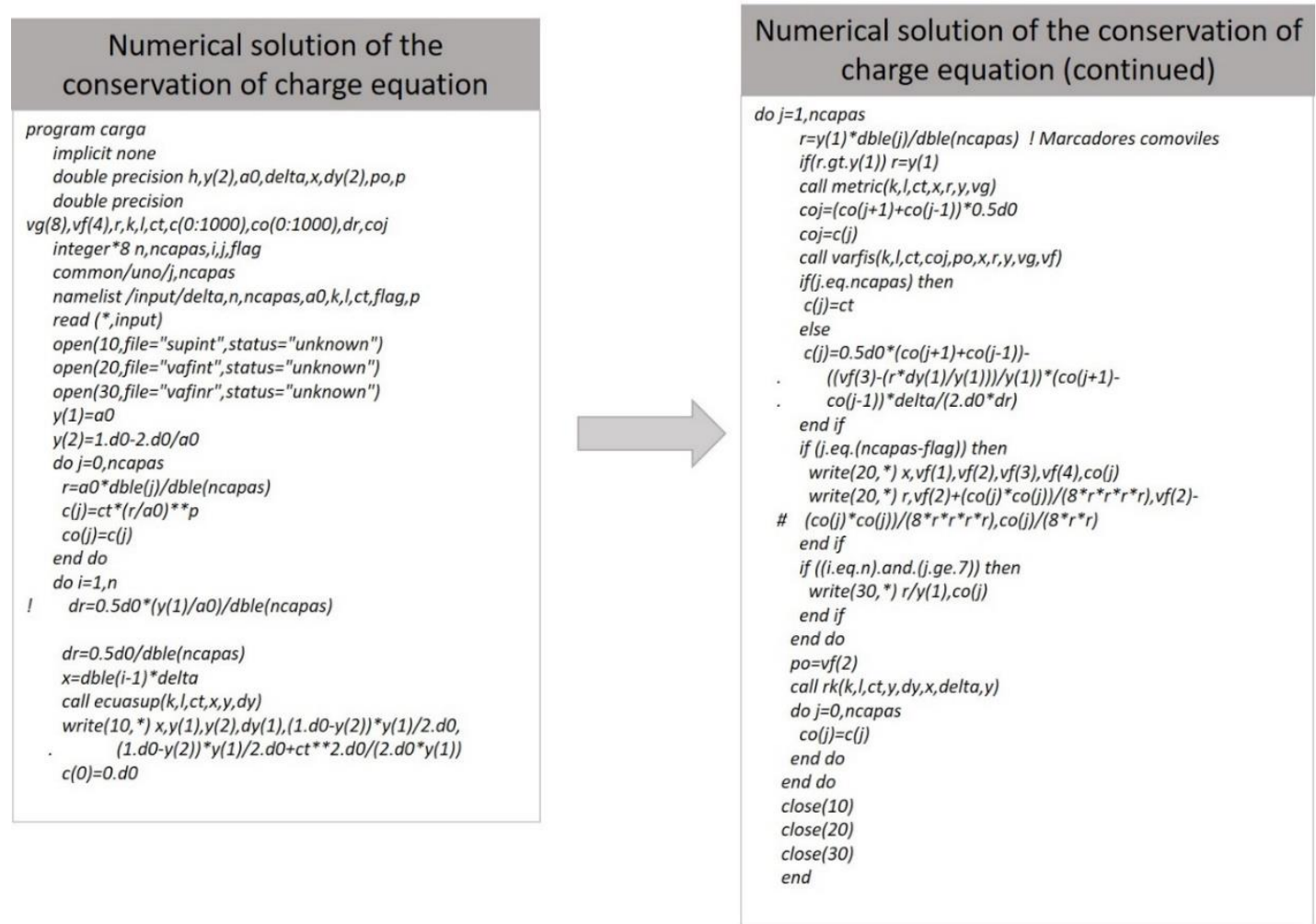

Figure 2. Numerical solution of the conservation of charge equation.

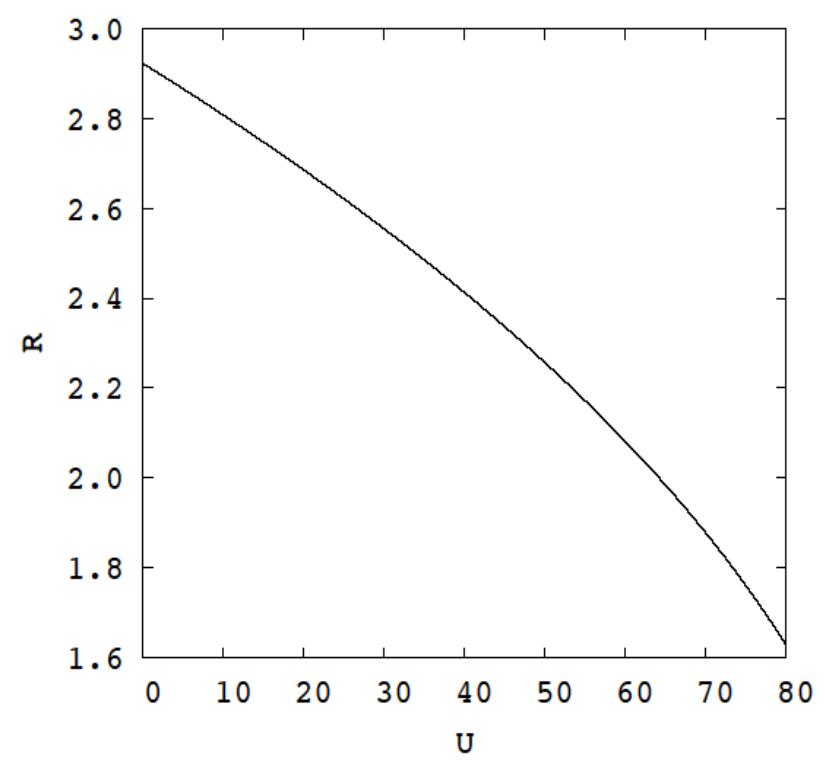

Figure 3. Rate of change of the distribution radius for constants $k=0.35 ; l=0.5$ and initial data of initial charge and radius $C=0: 292$ and $R(0)=2: 923$, respectively. 


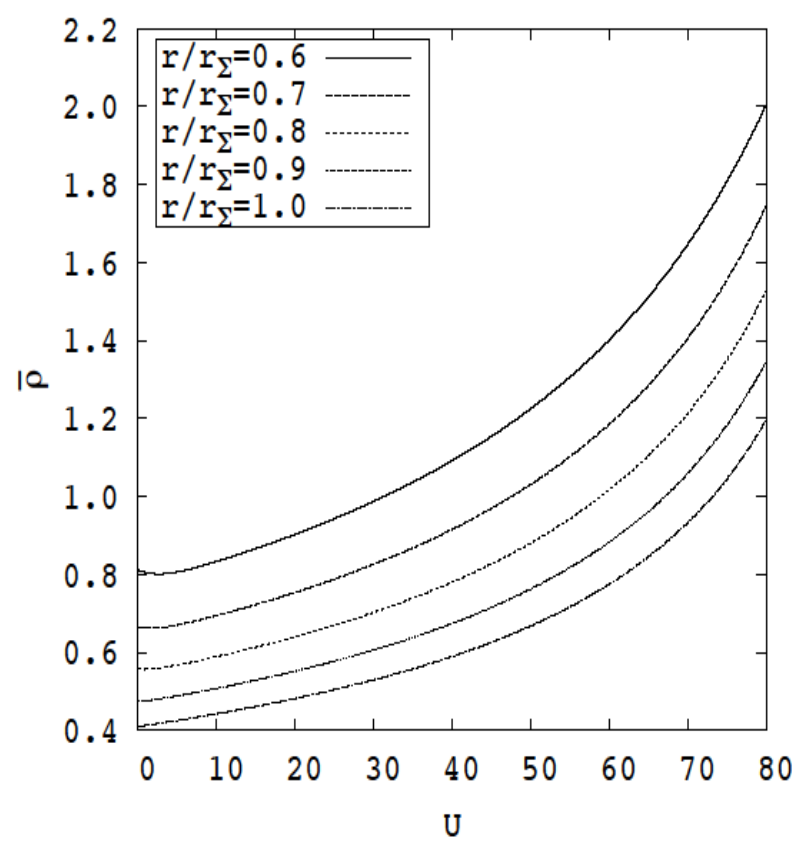

Figure 4. Changes in the matter density with time in different layers (multiplied by $10^{2}$ ).

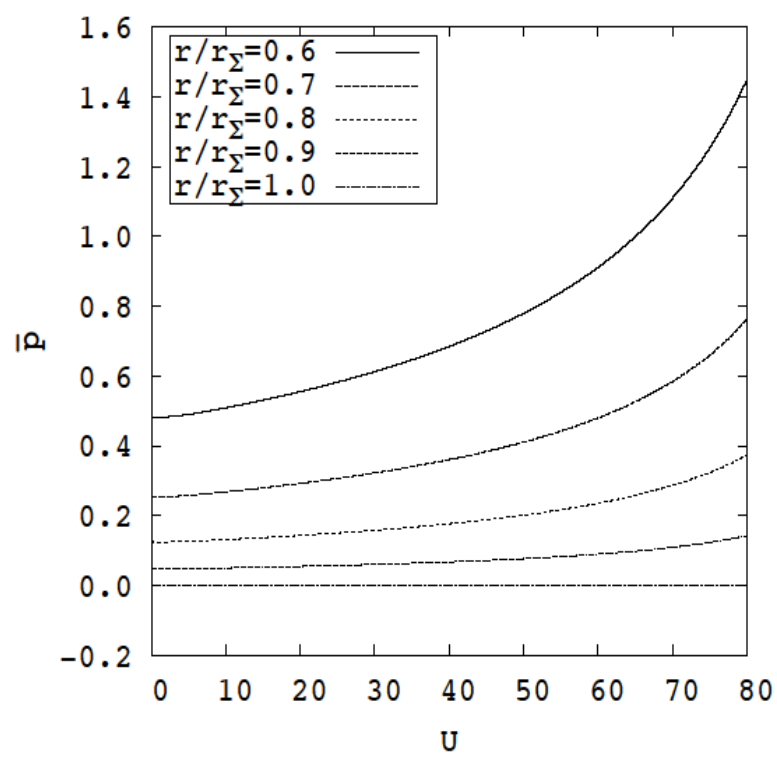

Figure 5. Evolution with time of the radial pressure (multiplied by $10^{2}$ ). 


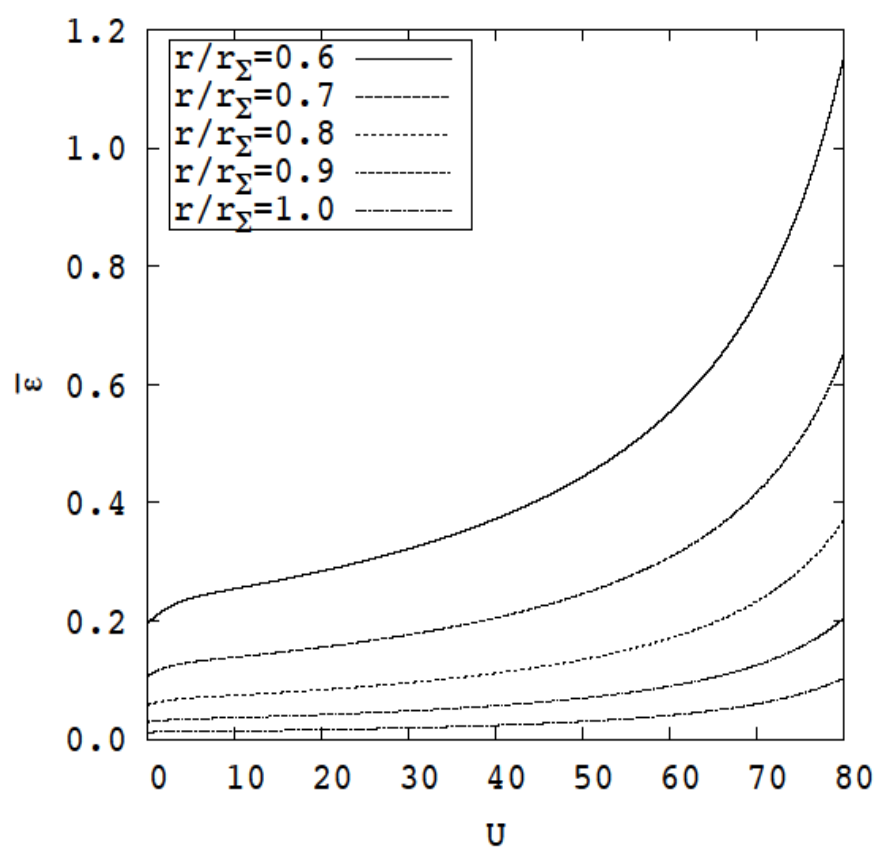

Figure 6. Radiation flux (multiplied by $10^{2}$ ).

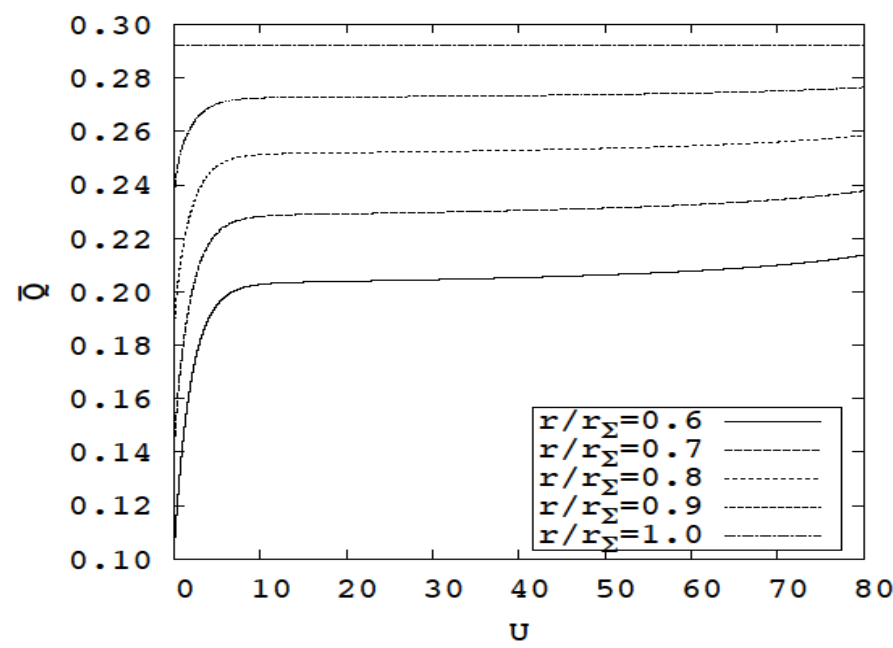

Figure 7. The changes are shown for different layers of the matter distribution.

\section{Conclusions}

An additional homothetic (self-similar) symmetry to space-time was assumed, and nonstatic solutions for the electromagnetic system with added gravity (Einstein-Maxwell systems) are obtained in a simple form. Coupling with the outer Reissner-NorstromVaidya space-time and using it as a transport mechanism, the radiation flux freely escapes from the distribution.

It has been demonstrated, once again, as well as matter can be considered as an anisotropic fluid, when it is electrically charged. The approach is achieved by obtaining a dynamical model by means of the free escape approximation and self-similarity within the source. It is represented in the chosen example by manipulating only the initial conditions $k, l$, and CT and setting physical conditions: $-1<\omega<1, p<\rho$ y $\rho>0$. A considerable set of initial conditions was selected for several runs of the programs, verifying the results shown. Several solutions were discarded, as they did not meet the above energy conditions.

More general solutions of the symmetry equations are needed to explore the generality of the results shown in this paper. Nevertheless, the anisotropic approach employed 
achieves results that are in agreement with those reported in other previously published work and which conclude that the final state is the steady-state [18].

An important feature is the anisotropy factor $p_{r}-p_{t}=2 \mu$, where it is clear that the loading induces anisotropy. If the charge is zero, of course, the anisotropy factor is zero. This fact was used as a way to validate the code, because results for zero charges have been reported previously. Figure 3 shows the radius of the distribution, which collapses inexorably. Unlike $[2,13,18,42,43]$, the electric charge does not stop the collapse.

In theory, while a black hole can possess a very large charge, the charge of the newly detected holes will be considered negligible. This assumption is based on the fact that a black hole is always surrounded by plasma. With the self-similar solutions proposed in this work, new models of black hole formation can be considered and also verify that the electric charge in the formation of a black hole can be significant. Models already reported establishing that, although the charge of a black hole is large, the charge/mass ratio is small and is not enough to significantly affect either the gravitational field of the matter distribution or the dynamics of the star or its collapse [44-53].

Author Contributions: Conceptualization, F.S.-C. and L.R.-R.; methodology, F.S.-C. and L.R.-R.; investigation, F.S.-C. and L.R.-R.; writing — original draft preparation L.R.-R.; writing-review and editing F.S.-C.; supervision, F.S.-C. and L.R.-R.; project administration, F.S.-C. and L.R.-R. All authors have read and agreed to the published version of the manuscript.

Funding: This research was funded by La Universidad de Las Américas-Ecuador and Universidad Politécnica de Venezuela, Puerto Ordaz.

Institutional Review Board Statement: Not applicable.

Informed Consent Statement: Not applicable.

Data Availability Statement: Not applicable.

Conflicts of Interest: The authors declare no conflict of interest.

\section{References}

1. Fujii, H.; Ueno, M.; Kobayashi, K.; Watanabe, M. Characteristic Length and Time Scales of the Highly Forward Scattering of Photons in Random Media. Appl. Sci. 2019, 10, 93. [CrossRef]

2. Bekenstein, J. Hydrostatic Equilibrium and Gravitational Collapse of Relativistic Charged Fluid Balls. Phys. Rev. 1971, 4, 2185-2190. [CrossRef]

3. Boero, E.; Dotti, G. Perturbaciones Axiales de Espacio-Tiempos Esféricamente Simétricos. Bachelor Thesis, Universitat Nacional de Córdova, Cordova, Spain, 2011; pp. 1-27.

4. Navarro, A.; Núñez, L.; Ruedas, H.; Sanabria-Gomez, J. Colapso Gravitacional Radiativo Esfericamente Simétrico en la Aproximación Poscuasiestática. Rev. Col. Física. 2008, 40, 210-213.

5. Usov, V. Electric fields at the quark surface of strange stars in the color-flavor locked phase. Phys. Rev. 2004, 70, 067301. [CrossRef]

6. Di Prisco, A.; Herrera, L.; Le Denmat, G.; MacCallum, M.A.H.; Santos, N.O. Nonadiabatic charged spherical gravitational collapse. Phys. Rev. D 2007, 76, 064017. [CrossRef]

7. Carr, B.J.; Coley, A.A. An asymptotic analysis of spherically symmetric perfect fluid self-similar solutions. Class. Quantum Gravity 2000, 17, 4339-4352. [CrossRef]

8. Coley, A.; Taylor, T. Gravitational collapse of spherically symmetric perfect fluid with kinematic self-similarity. Interna-tional. J. Mod. Phys. D 2002, 11, 155-186.

9. Carr, B.J.; Coley, A.A.; Goliath, M.; Nilsson, U.S.; Uggla, C. Critical phenomena and a new class of self-similar spherically symmetric perfect-fluid solutions. Phys. Rev. D 2000, 61, 081502. [CrossRef]

10. Carr, B.; Coley, A.; Goliath, M.; Nilsson, U.S.; Uggla, C. Infinite Kinematic Self-Similarity and Perfect Fluid Spacetimes Class. Quantum Gravity 2001, 33, 1863-1895.

11. Ray, S.; Espíndola, A.L.; Malheiro, M.; Lemos, J.P.S.; Zanchin, V.T. Electrically charged compact stars and formation of charged black holes. Phys. Rev. D 2003, 68, 084004. [CrossRef]

12. Malik, T.; López-Mobilia, R. A further exploration of the black hole entropy via the weyl tensor. Int. J. Mod. Phys. 2019, 2, 31.

13. Barreto, W.; Da Silva, A. Gravitational collapse of a charged and radiating fluid ball in the diffusion limit. Gen. Relativ. Gravit. 1996, 28, 735-747. [CrossRef]

14. Barreto, W.; Da Silva, A. Self-similar and charged spheres in the diffusion approximation. Class. Quantum Gravity 1999, 16, 1783-1792. [CrossRef]

15. Ivanov, B.V. Charged anisotropic matter with linear or nonlinear equation of state. Phys. Rev. D 2010, 82, 044052. 
16. Bonnor, W.; Wikramasuriya, S.B. Are very large gravitational redshifts possible? Mon. Not. R. Astron. Soc. 1975, 170, 643-649. [CrossRef]

17. Barreto, W.; Rosales, L. Self-similar and charged spheres in the free-streaming approximation. Gen. Relativ. Gravit. 2011, 43, 2833. [CrossRef]

18. Rosales, L.; Barreto, W.; Peralta, C.; Rodríguez-Mueller, B. Nonadiabatic charged spherical evolution in the postquasistatic approximation. Phys. Rev. 2010, 82, 084014. [CrossRef]

19. Barreto, W.; Rodríguez, B.; Rosales, L.; Serrano, O. Self-similar and charged radiating spheres: An anisotropic ap-proach. Gen. Relativ. Gravit. 2006, 39, 23-39. [CrossRef]

20. Komathiraj, K.; Maharaj, S. Tikekar superdense stars in electric fields. Math. Phys. 2007, 98, 042501. [CrossRef]

21. Komathira, K.; Mahara, S. Generalised compact spheres in electric fields. Gen. Relativ. Gravity 2007, $24,4513-4524$.

22. Zurek, W.H.; Page, D. Black-hole thermodynamics and singular solutions of the Tolman-Oppenheimer-Volkoff equation. Phys. Rev. D 1984, 29, 628-631. [CrossRef]

23. Sifiso, A.; Ngubelanga, S.; Maharaj, S.D.; y Subharthi, R. Relativistic stars with polytropic equation of state. Astrofísica y Ciencia Espacial 2015, 130, 211.

24. Debabrata, D.; Maxim, K.; Farook, R.; Saibal, R.; Guha, B. Study on charged strange stars in f(R,T) gravity. Eur. Phys. J. 2018, $2019,70$.

25. Sarkar, N.; Singh, K.N.; Sarkar, S.; Rahaman, F. Compact star models in class I spacetime. Theor. Phys. 2019, 79, 516. [CrossRef]

26. Ngubelanga, S.A.; Maharaj, S.D.; Ray, S. Compact stars with quadratic equation of state. Astrophys. Space Sci. 2015, 357, 74. [CrossRef]

27. Kileba Matondo, D.; Maharaj, S.D. Nuevos modelos compactos anisotrópicos cargados. Astrofísica Cienc. Espac. $2018,437,2016$.

28. Bondi, H. The contraction of gravitating spheres. Proc. R. Soc. Lond. 1964, 281, 39-48.

29. Busemann, H. The Foundations of Minkowskian Geometry. Comment. Math. Helv. 1950, 24, 156-187. [CrossRef]

30. Peralta, C.; Rosales, L.; Rodriguez-Mueller, B.; Barreto, W. Shear Viscosity in the post-cuasiestatica approximation. Phys. Rev. 2010, 81, 104021.

31. Barreto, W.; Peralta, C.; Rosales, L. Equation of state and limit transport mechanism in self-similar spheres. Phys. Rev. 1999, 59, 024008.

32. Barreto, W. The Post-Quasistatic Approximation as a test bed for Numerical. Phys. Rev. 2009, 79, 107502. [CrossRef]

33. Takisa, P.M.; Maharaj, S.D.; Takisa, P.M.; Maharaj, S.D. Some charged polytropic models. Gen. Relativ. Gravity 2013, 45, 1951-1969. [CrossRef]

34. Thirukkanesh, S.; Maharaj, S.D. Charged anisotropic matter with linear eequation of state. Class. Quant. Gravity 2008, 25, 235001. [CrossRef]

35. Nyonyi, Y.; Maharaj, S.D.; Govinder, K.S. New charged shear-free relativistic models with heat flux. Eur. Phys. J. C 2013, 73, 2637. [CrossRef]

36. Baushev, A.; Chardonnet, P. ELECTRIC CHARGE ESTIMATION OF A NEWBORN BLACK HOLE. Int. J. Mod. Phys. D 2009, 18, 2035-2045. [CrossRef]

37. Deruelle, N.; Sasaki, M.; Sendouda, Y. Junction Conditions in f(R) Theories of Gravity. Prog. Theor. Phys. 2008, 119, 237-251. [CrossRef]

38. Barreto, W.; Castillo, L.; Barrios, E. Bondian frames to couple matter with radiation. Gen. Relativ. Gravit. 2010, 42, 1845-1862. [CrossRef]

39. Misner, C.W.; Sharp, D.H. Relativistic Equations for Adiabatic, Spherically Symmetric Gravitational Collapse. Phys. Rev. 1964, 136, B571-B576. [CrossRef]

40. Barreto, W. The Order of Sir Bondi to appear. Interciencia 2009, 34, 361.

41. Nyonyi, Y.; Maharaj, S.D.; Govinder, K.S. Higher-dimensional charged shear-free relativistic models with heat flux. Eur. Phys. J. C 2014, 74, 2952. [CrossRef]

42. De Leon, J.P. Self-similar cosmologies in 5D: Spatially flat anisotropic models. Int. J. Mod. Phys. D 2009, 18, 743-762. [CrossRef]

43. Coley, A.A.; Tupper, B.O.J. Spherically symmetric anisotropic fluid ICKV spacetimes. Class. Quantum Gravity 1994, 11, 2553-2574. [CrossRef]

44. Barreto, W.; Castillo, L.; Barrios, E. Central equation of state in spherical characteristic evolutions. Phys. Rev. D 2009, 80, 084007. [CrossRef]

45. Tupper, B.O.J. The equivalence of electromagnetic fields and viscous fluids in general relativity. J. Math. Phys. 1981, 22, 2666. [CrossRef]

46. Carr, B.J.; Yahil, A. Self-similar perturbations of a Friedmann universe. Astrophys. J. 1990, 360, 330-342. [CrossRef]

47. Gundlach, C. Critical gravitational collapse of a perfect fluid: Nonspherical perturbations. Phys. Rev. D 2002, 65, 084021. [CrossRef]

48. Barreto, W.; Rodríguez, B.; Martíneznez, H. Radiating Fluid Spheres in the Effective Variables Approximation. Astrophys. Space Sci. 2002, 282, 581-593. [CrossRef]

49. Ray, S.; Espindola, A.L.; Malheiro, M.; Lemos, J.; Zan hin, V.T. Electrically charged compact stars. Phys. Rev. 2003, 68, 084004.

50. Ray Int, S. Dark energy models with a time-dependent gravitational constant. Int. J. Mod. Phys. D 2007, 16, 1791-1802.

51. Matondo, D.K.; Maharaj, S.D. New charged anisotropic compact models. Astrophys. Space Sci. 2016, 361, 221. [CrossRef] 
52. Liang, M.; Lsn, Y. Spatiotemporally periodic solutions by variational methods. Sch. Phys. 2003. Available online: http: / / www.chaosbook.org/projects/Liang/Liang.pdf (accessed on 12 December 2003).

53. Antón, L.; Marti, J.; Miralles, J. Magnetohidrodinámica Relativista Numérica: Aplicaciones en Relatividad Especial y General. Ph.D. Thesis, Universitat de Valencia, Valencia, Spain, 2008. Available online: https://roderic.uv.es/handle/10550/14910 (accessed on 12 December 2003). 